\title{
2009 Año internacional de las fibras naturales, problemática y tecnología de irradiación
}

\author{
2009 International year of natural fibers, problematic and \\ irradiation technology
}

Johnny Vargas ${ }^{a}$, Flor Quispe ${ }^{b}$

\begin{abstract}
RESUMEN
En los últimos años las fibras sintéticas han desplazado a las fibras naturales, a pesar de sus ventajas para el medio ambiente, la salud y la seguridad alimentaria. La FAO ha elegido 2009 como el Año Internacional de las Fibras Naturales. La idea es incentivar la producción de las fibras naturales como el algodón, la lana de alpaca, pero al concientizar una mayor producción de fibras naturales como el algodón, esta genera 2 problemas, como son el mayor uso de pesticidas, perjudiciales para la salud, el ambiente y la resistencia de las plagas a los pesticidas, en este estudio analizamos las bondades de la tecnología de irradiación, como alternativa a la solución de estos problemas, tanto en el ámbito internacional y nacional, ya sea mediante la mutación inducida por radiación gamma para la obtención de nuevas variedades mejoradas y en la lucha contra las plagas utilizando la irradiación como tratamiento cuarentenario, siendo una alternativa viable como medida fitosanitaria para el control de plaga de insectos o mediante la Técnica del Insecto Estéril, (TIE) para la erradicación de plagas, reemplazando fumigantes químicos perjudiciales para la salud y el medioambiente. En Pakistán un cultivar de mutantes de alto rendimiento en algodón, inducidos mediante el uso de rayos gamma, ha aportado más de 3000 millones de dólares. En el Perú a través del IPEN se ha irradiado semillas de algodón para la obtención de variedades mejoradas, apoyando a la Asociación de Agricultores de Cañete y a la Universidad Pedro Ruiz Gallo de Lambayeque. También con la tecnología de irradiación se esteriliza productos de algodón de uso médico como gasa, gasa parafinada, hisopos y en la industria cosmética la esterilización de brochas elaboradas de finas fibras de animales. En la fibra de lana de alpaca, el IPEN también contribuye conjuntamente con la Universidad Cayetano Heredia a mejorar la producción de fibra fina. La tecnología de irradiación genera nuevas variedades mejoradas, para aumentar la producción del algodón, resistir el ataque de plagas, como tratamiento cuarentenario puede solucionar problemas fitosanitarios y mediante la técnica del insecto estéril, elimina plagas reemplazando pesticidas perjudiciales para la salud y el ambiente, acrecentando la calidad de los productos agroexportables.
\end{abstract}

Palabras clave: Radiación gamma, fibras naturales, algodón, lana de alpaca, mutación, esterilización, pesticidas, plagas

\begin{abstract}
In recent years the synthetic fibers have displaced natural fibers, despite its advantages for the environment, the health and the food safety. FAO has chosen 2009 as the International Year of Natural Fibers. The idea is to encourage the production of natural fibers such as cotton, wool, alpaca, but a greater awareness to the production of natural fibers like cotton, this creates 2 problems, such as increased use of pesticides harmful to health, the environment and the resistance of pests to pesticides, this study analyzed the benefits of the technology of irradiation as an alternative to solve these problems, at both international and national, either by mutation induced by gamma radiation to obtain new improved varieties and pest control using irradiation as a quarantine treatment is a viable alternative crop as a measure to control pest insects or the Sterile Insect Technique, (SIT) for the eradication of pests, replacing harmful chemical fumigants for health and the environment. In Pakistan a cultivar of mutant cotton yield, induced by the use of gamma rays has contributed more than 3000 million dollars. In Peru, IPEN is irradiated cotton seeds to obtain improved varieties, supporting the Association of Farmers of Cañete and University Pedro Ruiz Gallo of Lambayeque. Also with the technology of irradiation improves the quality of cotton products for medical use such as gauze, swabs and cosmetic brushes sterilization of fine fibers produced from animals. In alpaca fiber, IPEN also helps with the Cayetano Heredia University to improve the production of fine fiber. Irradiation technology creates new improved varieties to increase production of cotton or resist attack by pests, such as quarantine treatment plant and can solve problems through the sterile insect technique eliminates replacing pesticides pests harmful to health and the environment, increasing the quality of agro-exporting products.
\end{abstract}

Keywords: gamma radiation, natural fibers, cotton, alpaca, transfer, sterilization, pesticides, pests

a Instituto Peruano de Energía Nuclear

b Probuysa EIRL 


\section{INTRODUCCIÓN}

En los últimos años las fibras sintéticas han desplazado a las fibras naturales, a pesar de sus ventajas para el medio ambiente, la salud y la seguridad alimentaria.

La Organización de las Naciones Unidas para la Agricultura y la Alimentación (FAO) ha elegido 2009 como el Año Internacional de las Fibras Naturales (AINF). Su objetivo es concienciar a los consumidores de la importancia de estos cultivos para el medio ambiente, la salud y la seguridad alimentaria de todo el planeta. Cada año se producen unos 30 millones de toneladas de dichas fibras en todo el mundo. Sin embargo, su consumo ha sido sustituido progresivamente desde los años 60 por los materiales sintéticos. Por ello, otro de los objetivos principales del AINF pasa por promover su producción y consumo [1].

Los responsables de la FAO ofrecen varias razones para promocionar estos productos naturales, en concreto 15 elementos de origen vegetal y animal que se producen tradicionalmente en todo el mundo. Su producción supone una importante fuente de ingresos: se trata de un sector que genera para los agricultores de todo el mundo unos 29.000 millones de euros anuales.

El valor económico de las fibras es especialmente elevado en los países en desarrollo, donde puede alcanzar en algunos casos hasta la mitad de las exportaciones. Por lo tanto la seguridad alimentaria de millones de personas depende de las fibras naturales.

Desde el decenio de 1960 ha aumentado el uso de fibras sintéticas, y las fibras naturales han perdido participación en el mercado. Los productores y fabricantes afrontan el reto de crear y mantener los mercados en los que puedan competir con eficacia contra las fibras sintéticas. En algunos casos esto ha significado definir y promover nichos de mercado. En otros, donde las ventajas naturales les permiten competir con eficacia contra las fibras sintéticas, se requieren investigación básica y desarrollo para facilitar el uso de las fibras naturales en aplicaciones nuevas [2].

Las fibras naturales son recursos renovables, reemplazarían a las fibras sintéticas o artificiales, que son producidos a partir de sustancias del petróleo.
Además el uso de estos tejidos naturales no causa alergias sobre la piel cuando, son usados como vestimentas o utilizados con otros propósitos, la idea es que halla una producción sostenida, que beneficie a los productores, agricultores, pastores, la industria, los consumidores y el medioambiente.

Las fibras naturales son más saludables (algodón, lana de alpaca, etc.), fresca en verano y protege en el frío, además son renovables, reemplaza a las fibras sintéticas derivadas del petróleo, son biodegradables, los

cultivos neutralizan el cambio climático, son fuente de trabajo y mejora de la economía de millones de agricultores y pastores que viven de las fibras naturales.

\section{El Problema : Pesticidas}

Por otro lado, la FAO también recuerda que el cultivo insostenible de estos materiales conlleva una serie de perjuicios.

Por ejemplo, sus responsables destacan el caso de la producción intensiva del algodón, que ha supuesto el uso masivo de pesticidas, principalmente insecticidas, con un riesgo serio para la salud de granjeros, consumidores, la agrobiodiversidad, el agua potable y los ecosistemas.

El uso de plaguicidas de reconocida peligrosidad, así como el mal uso de estos productos en general, viene dejando secuelas negativas, muchas veces de carácter irreversible, tanto sobre el ser humano como en el ambiente.

\section{¿A donde van a dar los plaguicidas?}

De acuerdo con las investigaciones realizadas por el Departamento de Agricultura de los EE.UU., entre $97 \%$ y $99 \%$ de las cantidades de los plaguicidas aplicados no alcanzaron los organismos que se desean combatir (ERF, 1991), citado por [3].

Esto quiere decir que de 1000 millones de toneladas de pesticidas utilizadas en los Estados Unidos, solo el $1 \%$ al $3 \%$ alcanzan a las plagas, el resto se disipa en el ambiente.

Según datos de la OMS anualmente se intoxican 2 millones de de personas por 
exposición directa o indirecta a plaguicidas. Con un resultado de más de 200000 muertes.

El $75 \%$ de estos hechos ocurren en países en desarrollo, donde únicamente se utiliza el 25 $\%$ de la producción mundial de plaguicidas.

Se estima que más de 700000 personas al año sufren los efectos crónicos.

Desde el año 1991 se han prohibido el uso de algunos fumigantes aplicados a los alimentos, según la Fig. 1 los fumigantes han sido prohibidos por afectar seriamente la salud, como el óxido de etileno y el dibromuro de etileno, este último fue reemplazado por el bromuro de metilo que resulta ser peor, ya que no solamente es perjudicial para la salud sino que es 50 veces más destructor de la capa de ozono que los clorofuorocarbonados y al hablar de la destrucción de la capa de ozono es hablar de la destrucción del Planeta.

Es una triste historia la aplicación de los fumigantes químicos en la agricultura y la alimentación :

El óxido de etileno, se utilizó para la descontaminación microbiana de especias como la pimienta, actualmente su uso se limita a la esterilización de algunos materiales médicos. El óxido de etileno es cancerígeno. Desde 1991, la Unión Europea (UE) ha prohibido el empleo del óxido de etileno y el óxido de propileno para destruir los microorganismos presentes en las especias y condimentos secos para alimentos, debido a preocupaciones relacionadas con la carcinogénesis y la seguridad de los trabajadores [4].

El dibromuro de etileno, conocido por sus siglas en inglés (EDB) fue usado para la eliminación de insectos en cereales y en el tratamiento cuarentenario de frutas y vegetales, su uso fue prohibido en los Estados Unidos en 1984. Es genotóxico y carcinógeno.

El bromuro de metilo es un gas tóxico, daña el SNC, También se ha identificado al BrM como una de las sustancias que agota la capa de ozono, dejando pasar los rayos ultravioletas dañinos (UV-B). Si la capa de ozono no reduce esta radiación ultravioleta, el ecosistema marítimo, la producción agrícola, los animales se verían afectados y aumentaría la incidencia de cáncer.

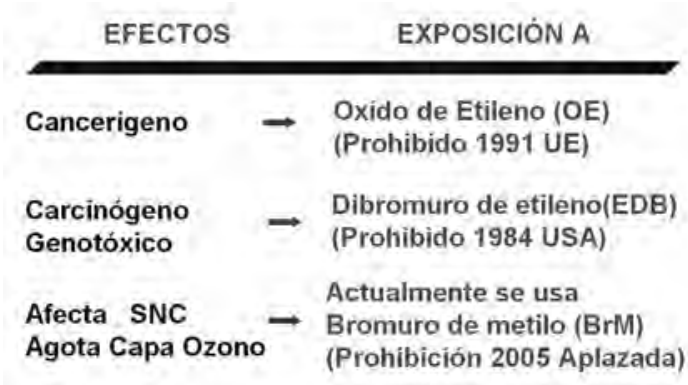

Figura 1. Fumigantes prohibidos para alimentos (J. Vargas)

La Agencia de Protección Ambiental de EE.UU. (EPA) por sus siglas en inglés, está fortaleciendo medidas de seguridad para pesticidas fumigantes de suelos. Las medidas de seguridad reducirán las exposiciones a fumigantes entre personas circundantespersonas que viven, trabajan, asisten a la escuela, o pasan tiempo cerca de campos agrícolas que son fumigados-y aumentarán la seguridad general del uso de fumigantes al requerir una mayor planificación y cumplimiento.

\section{El Problema: Plagas}

Además, las plagas se vuelven cada vez más resistentes a los pesticidas, lo que lleva a los agricultores a aumentar su uso, y con ello su impacto económico, ambiental y social. (Figura 2)

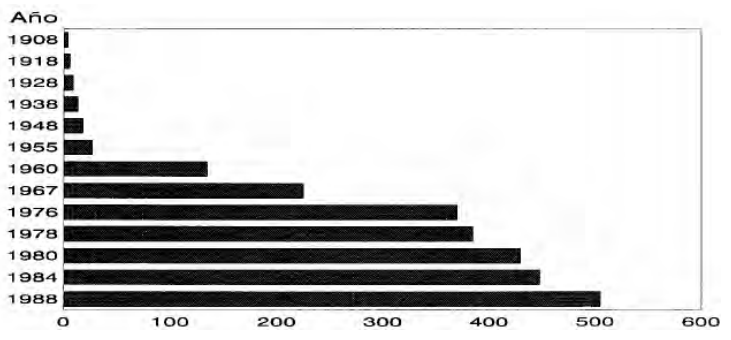

Figura 2. Especies de plagas resistentes a plaguicidas (PNUMA)

Por otra parte, la introducción de variedades de algodón mejoradas e híbridos para mejorar el rendimiento ha supuesto también incrementar el uso de insecticidas, ya que son más susceptibles a las plagas que los cultivos tradicionales.

El problema del desarrollo de resistencia en los insectos y plantas atacadas por el pesticida 
ha adquirdo magnitudes alarmantes. Hoy es corriente ver que una maleza suceda a otra, 0 que un insecto reemplace a otro o, por último que una especie desarrolle resistencias a determinados compuestos tóxicos en el ambiente natural $v$ han creando mecanismos de adaptación en insectos, malezas y bacterias.

En el valle de Cañete en Perú, el proceso se estudió por un periodo de veinte años en los cultivos de algodón. A partir de 1949, se empezaron a utilizar hidrocarburos clorados, y su aplicación redundó en aumentos importantes de productividad $(20 \%)$ pero, con la eliminación de los controles biológicos naturales, aparecieron nuevas plagas $\mathrm{y}$, al mismo tiempo, las pestes atacadas desarrollaron resistencias especialmente al DDT y al dieldrin. La respuesta fue una utilización más intensiva de fertilizantes con el único resultado de aumentar violentamente los costos en circunstancias que la productividad se reducía a niveles inferiores al de 1949 [5].

Por otro lado el Grupo Intergubernamental de Expertos sobre el Cambio Climático, IPCC, ha determinado que el cambio climático tendrá graves consecuencias en los ecosistemas mundiales, en la agricultura, los bosques, la salud humana y el desarrollo de plagas.

\section{APLICACIONES DE LA TECNOLOGÍA DE IRRADIACIÓN EN FIBRAS NATURALES}

\section{Obtención de nuevas variedades mejoradas inducidas por radiación}

Según Slavko Borojevic (1984), la aparición de mutaciones espontáneas son ínfimas alrededor de 1 en un millón; sin embargo, la tasa de aparición de mutaciones inducidas puede incrementarse hasta 1 en 100.

En el libro Crop Variety Improvement and Its Effect on Productivity 2003, el porcentaje de arrozales dedicados a variedades producidas con ayuda de la irradiación en 1998 fue de al menos $28 \%$ en Tailandia, $19 \%$ en Lao y $14 \%$ en Vietnam. En el Japón, los investigadores estiman que las variedades vegetales producidas mediante mutaciones inducidas por radiaciones tienen una participación en el mercado de 804 millones de dólares según se informó en el Journal of Nuclear Science and Technology (Octubre 2002).

En Pakistán, el 25\% de la plantación de la zona algodonera está constituida por un cultivar de mutantes de alto rendimiento inducidos mediante el uso de rayos gamma. Se estima que ese cultivar ha aportado más de 3000 millones de dólares en producción algodonera [6].

En el Perú, en el marco de un convenio de cooperación entre el IPEN y la Estación experimental Agrícola de la Asociación de Agricultores de Cañete (AACñ), se están realizando trabajos de investigación en forma conjunta con el fin de inducir mutaciones a semillas de algodón mediante irradiación gamma y generar una nueva variedad de algodón con valor agregado ya sea en la productividad y/o calidad del algodón Tangüis poniendo énfasis en la búsqueda de una variedad de algodón de mayor precocidad con un periodo vegetativo menor a 210 días [7].

También el IPEN a fines del 2008 apoyó un proyecto de tesis de la Universidad Nacional «Pedro Ruíz Gallo» titulado «Mejoramiento del cultivo de Algodón por Medio de Irradiación Gamma en la Provincia de Lambayeque»

Dosis menores a $0.5 \mathrm{kGy}$ son ideales para conseguir diferentes efectos en los cultivares como por ejemplo mayor rendimiento, resistencia a plagas y enfermedades, mayor precocidad, resistencia a las heladas, resistencia a la salinidad, mayor finura del hilo del algodón, etc.

Si logramos obtener variedades resistentes a plagas se evitaría el uso de pesticidas.

\section{LUCHA CONTRA LAS PLAGAS}

Por radiaciones se pueden eliminar insectos como moscas, gorgojos y polillas en cualquier fase o estadío (huevo, larva, pupa o insecto adulto) en granos, cereales, harinas, hierbas aromáticas, frutos y frutas secas, otros productos de almacenamiento o exportación en bodegas de los barcos reemplazando fumigantes químicos perjudiciales para la salud y el medio ambiente, con la ventaja que la radiación gamma es un proceso físico y de mayor eficacia. 


\section{Tratamiento cuarentenario}

El comercio internacional de productos agrícolas obliga al uso de tratamientos cuarentenarios para evitar el ingreso de plagas foráneas, práctica que es demandada por los países importadores como EE.UU. y Japón, dos de los países, que más frutas y vegetales importan del mundo.

Productos peruanos como mangos y espárragos, antes de ingresar al mercado de USA, son obligados al tratamiento fitosanitario cuarentenario, como el hidrotérmico y la fumigación con Bromuro de Metilo (BrM).

El tratamiento hidrotérmico se realiza en Piura para los mangos de exportación, para el control de la Mosca de la Fruta o Mosca del Mediterráneo (Ceratitis capitata), con la aprobación del Servicio de Inspección y Salubridad de Plantas y Animales (APHIS) del Departamento de Agricultura de los Estados Unidos (USDA). Este tratamiento con agua caliente demanda mayor costo, consumo de energía, contaminación ambiental, tiempo y mantenimiento y puede alterar las características físico-químicas, nutricionales y la calidad sensorial del mango. [8]

Para el caso del espárrago peruano el tratamiento cuarentenario es la fumigación con BrM, para el control de la polilla (Copitarsia decolora). Este fumigante reemplazó al dibromuro de etileno, prohibido en los Estados Unidos desde 1984.

El Bromuro de Metilo es perjudicial para la salud y es 50 veces más destructor de la capa de ozono que los clorofluorocarbonos. Los 183 países suscriptores del Protocolo de Montreal (1997) acordaron ir reduciendo progresivamente el uso del BrM, hasta su prohibición total en el 2005. En la décima sexta reunión de las Partes del Protocolo de Montreal sobre las sustancias que agotan la capa de ozono que se realizó en Praga en Noviembre del 2004, se acordó que 11 naciones desarrolladas, incluyendo Estados Unidos, podrán seguir utilizando el BrM, puesto que no existen alternativas que sean económicamente factibles.

Por consiguiente, se ha aplazado la fecha límite para la eliminación total del BrM debido a que no se han encontrado alternativas útiles con las características que se necesitan [9].
En el Perú se han realizado trabajos de investigación utilizando la tecnología de irradiación, como alternativa cuarentenaria contra las plagas reemplazando tratamientos costosos que alteran las características intrínsecas del producto o fumigantes perjudiciales para la salud y el medioambiente, casos concretos son los mangos (contra la mosca Ceratitis capitata) y espárragos (contra la polilla Copitarsia decolora).

Debido a estas perspectivas, la Tecnología de Irradiación surge como una alternativa viable, como tratamiento cuarentenario y ser una medida fitosanitaria para sus frutas y hortalizas de exportación, en reemplazo de métodos convencionales que utilizan fumigantes nocivos para la salud y el medio ambiente.

\section{Erradicación de plagas}

La técnica del insecto estéril (TIE) o control autocida consiste en traer del campo al laboratorio a una plaga, reproducirla en grandes cantidades, esterizarlas mediante radiación gamma para que no se puedan reproducir, enviarlas de vuelta al campo y liberarlas en forma masiva, con la finalidad de que se crucen con las moscas nativas; de tal manera, que cuando más cruzamiento haya entre ambas, se obtenga como resultado una menor reproducción.

Esta técnica se ha usado exitosamente para erradicar plagas en países como Estados Unidos, México, Chile, Argentina y el Perú. En nuestro país, el SENASA ha logrado erradicar la mosca de la fruta Ceratitis capitata en Moquegua y Tacna, la idea es que mediante la tecnología de irradiación se pueda erradicar esta plaga, y otras, en toda la Costa, con la finalidad de hacer factible la exportación de más de una veintena de especies vegetales con restricciones fitosanitarias.

\section{OTRAS APLICACIONES DE LA TECNOLOGÍA DE IRRADIACIÓN EN LAS FIBRAS NATURALES}

\section{Material Médico}

Dosis de radiación gamma entre 15 y 25 kGy produce la esterilización en productos de algodón de uso médico, como copos de 
algodón, gasa, gasa parafinada para heridas de quemaduras, hisopos, hisopos vaginales, etc.

\section{Material cosmético}

La industria cosmética es muy exigente en la calidad de sus insumos y producto terminado, por lo que la tecnología de irradiación se aplica en la descontaminación de brochas cosméticas elaboradas de fibras finas naturales de animales.

\section{Fibra de Alpaca}

En el Perú no existe un programa de mejoramiento genético en alpacas basado en el uso de de marcadores genéticos para la selección y reproducción de animales que garanticen la obtención de crías con buena calidad de fibra.

Por ello es importante que las técnicas de selección sean asistidas mediante el uso de marcadores moleculares para tener un sistema de registro confiable como una herramienta que permitan acelerar los resultados esperados en los programas de mejoramiento del país.

La Unidad de Biotecnología Molecular (UBM) de la Universidad Peruana Cayetano Heredia conjuntamente con el Instituto Peruano de Energía Nuclear (IPEN) están colaborando para solucionar este problema, por lo que han elaborado una prueba para determinación de parentesco e identificación en alpacas usando tecnología de punta de acorde a los estándares internacionales [10].
También en el IPEN, se ha desarrollado y construido un sistema para medir el tamaño del grosor de la fibra de alpaca. El sistema esta basado en el análisis de una imagen 240x de la fibra, el sistema que toma la imagen ya esta completamente implementado, es portátil y autónomo [11].

\section{CONCLUSIONES}

La radiación gamma garantiza la inocuidad y la conservación de los productos agroexportables permitiendo su llegada a mercados distantes.

La mutación inducida por radiación gamma, permite obtener nuevas variedades mejoradas, acrecentando la producción y calidad de los productos de los cultivos.

La radiación gamma es una alternativa que ya ha demostrado ser exitosa como tratamiento cuarentenario para la agroexportación.

La tecnología de irradiación a través del tratamiento cuarentenario y la técnica del insecto estéril, es un proceso eficaz en la lucha contra las plagas reemplazando fumigantes o pesticidas perjudiciales para la salud y el medio ambiente.

Las investigaciones que realizan el IPEN y otras entidades públicas y privadas en fibras naturales beneficiarán a los agricultores de algodón y alpaqueros que viven gracias a estas fibras.

La Tecnología de Irradiación permitirá ampliar y mejorar el acceso de los productos peruanos al exigente mercado internacional.

\section{REFERENCIAS BIBLIOGRÁFICAS}

[1]. Fernández A. Porque hay que proteger las fibras naturales. CONSUMER EROSKI. [ en línea] 28 de Mayo de 2009 [ fecha de acceso 1 de Junio de 2009 ];URL disponible en : http://www.consumer.es/web/es/medio ambiente/ naturaleza/2009/05/28/185603.php

[2]. AIFN_hojas.pdf. EL AÑO INTERNACIONAL DE LAS FIBRAS NATURALES 2009. ¿ Por qué naturales? URL disponible en: www.naturalfibres2009.org

[3]. García E. Consecuencias indeseables de los plaguicidas en el ambiente. AGRONOMÍA MESOAMERICANA 8 (1) 119 -135 [en línea].1997. [fecha de acceso 1 de Mayo de 2009]; URL disponible en: http://www.mag.go.cr/ rev meso/v08n01 120.pdf

[4]. Loaharanu P. Creciente demanda de alimentos inocuos. Boletín del OIEA. 2001; 43 (2): 37 - 42

[5]. Bifani P. Medio Ambiente y Desarrollo Sostenible. 1999 : 352

[6]. IAEA International Atomic Energy Agency. Bulletin Volume $45 \mathrm{~N}^{\circ} 2$ - Looks at the legacy of «Atoms for Peace». December 2003. 
[7]. Montoya Y. et al. Investigaciones con mutaciones para mejorar la productividad y calidad del algodón peruano. Tecnología \& Desarrollo. Diciembre 2006; 2 (3): 19 - 22.

[8]. Vargas J. Tecnología de Irradiación y Agroexportación. Revista Industria Alimentaria. 2008; (6): 12 -15

[9]. Mejía M. Boletín de la NAPPO. Organización Norteamericana de Protección a las Plantas. Marzo de 2005.

[10]. Espinoza JR. et al. Genómica de Alpacas: Identificación de genes expresados y marcadores genéticos asociados a la Productividad de fibra fina en alpacas [en línea]. 2007. [fecha de acceso 1 de julio de 2009]; URL disponible en: http://www.alpacafiestaperu.com/event1002.html

[11]. Baltuano O. et al. Prototipo de Fibrómetro Digital Computarizado para Medición Automática del espesor de Fibra de Alpaca. Informe Científico Tecnológico 2005; 105 -113

E-mail: jvargas@ipen.gob.pe 